\title{
A quaternion-based attitude tracking controller for robotic systems
}

\author{
By James D. Biggs \\ Department of Mechanical \& Aerospace Engineering, University of Strathclyde.
}

\begin{abstract}
This paper presents a new quaternion-based attitude tracking controller. A general Lyapunov function is defined whose derivative is control dependent and a control is chosen to guarantee asymptotic stability of the zero-error state. The corresponding closedloop error dynamics are shown to reduce to a simple 1 degree of freedom description in terms of the eigen-axis angle error. The main contribution of this paper is to present a special case where the closed-loop error dynamics reduce to a simple linear oscillator description (without the need for linearisation). This means that the controller can be tuned to guarantee exponentially fast tracking with a damped response and without oscillation.
\end{abstract}

\section{Introduction}

Quaternion-feedback control has been used to control the attitude of many robotic systems such as spacecraft, see Wie, B., Weiss, H.,Arapostathis, A. (1989), Wen, J. T.,Kreutz-Delgado, K., (1991), Wie, B., Barba, P. M. (1985), autonomous underwater vehicles, see Fjellstad, O. E.,Fossen, T. I., (1994) and quadrotor vehicles, see Tayebi, A., McGilvray, S., (2006). In this paper a new quaternion-based tracking controller where the zero-error state of the closed-loop error system is globally asymptotically stable is presented. Although other quaternion-based tracking controls have been developed, the closed-loop system error dynamics of the presented control reduces to a linear description of the rotation angle error. This paper uses a general discontinuous Lyapunov function similar to that proposed in Fragopoulos, D., Innocenti, M., (2004) where it was used to develop a stabilisation control and which is naturally extended here to a tracking controller. The time derivative of this Lyapunov function is control dependent and a control is chosen to asymptotically stabilize the zero-error attitude state of the closed-loop system. The main result in this paper is to present a special case where the closed-loop equations reduce to a linear description of the tracking error without the need for approximations or linearisation. The important feature of this is that the control gains can be easily tuned to drive this tracking error to zero exponentially fast and yielding a damped response without oscillations. 


\section{Attitude dynamics and kinematics}

The dynamic equations of motion for a controlled rigid body without external disturbances are described by (see for example Hughes, P. C., (2004))

$$
J \dot{\omega}=J \omega \times \omega+\mathbf{u},
$$

where $J$ is a known positive definite, symmetric inertia tensor, $\omega=\left[\omega_{1}, \omega_{2}, \omega_{3}\right]^{T}$ is the angular velocity vector and $\mathbf{u}$ is the applied torque. In practise there will be external disturbance torques which are often negligible, such as with spacecraft. The kinematics described in quaternion form are

$$
\begin{aligned}
& \frac{d \mathbf{q}}{d t}=\frac{1}{2} \hat{\Omega} \mathbf{q}+\frac{1}{2} q_{4} \omega \\
& \dot{\dot{q}_{4}}=-\frac{1}{2} \omega^{T} \mathbf{q},
\end{aligned}
$$

where $\bar{q}=\left[q_{1}, q_{2}, q_{3}, q_{4}\right]^{T}$ is the current state quaternion which can be expressed as $\bar{q}=\left[\mathbf{q}, q_{4}\right]^{T}$ with $\mathbf{q}=\left[q_{1}, q_{2}, q_{3}\right]^{T}$ with the inverse quaternion defined by $\bar{q}^{-1}=\left[-\mathbf{q}, q_{4}\right]^{T}$ and where $\hat{\Omega}$ is an element of the Lie algebra of the rotation group $\mathrm{SO}(3)$ whose Lie bracket is defined by $[X, Y]=X Y-Y X$. $\hat{\Omega}$ is related to the angular velocity vector $\omega$ via the "hat" map which maps isomorphically an element of a vector in Euclidean space $x \in \mathbb{R}^{3}$ to an element of the Lie algebra of $\mathrm{SO}(3), \hat{\therefore}: \mathbb{R}^{3} \rightarrow \mathfrak{s o}(3)$

$$
S(x)=\left(\begin{array}{ccc}
0 & -x_{3} & x_{2} \\
x_{3} & 0 & -x_{1} \\
-x_{2} & x_{1} & 0
\end{array}\right) .
$$

A rotation matrix can then be retrieved from $\bar{q}$ as:

$$
R(\bar{q})=I_{3 \times 3}+2 q_{4} S(\mathbf{q})+2 S(\mathbf{q})^{2},
$$

where $I_{3 \times 3}$ is a $3 \times 3$ identity matrix. The kinematics $(2.2)$ can be expressed equivalently as $\dot{R}(\bar{q})=R(\bar{q}) \hat{\Omega}$. The problem is then to track a commanded (in general time-dependent) quaternion $\bar{q}_{c}=\left[q_{1 c}, q_{2 c}, q_{3 c}, q_{4 c}\right]^{T}$ and angular velocity $\omega_{c}$ that define a feasible attitude $\dot{R}\left(\bar{q}_{c}\right)=R\left(\bar{q}_{c}\right) \hat{\Omega}_{c}$. The error quaternion $\bar{q}_{e}=\left[\mathbf{q}_{e}, q_{4 e}\right]$ where $\mathbf{q}_{e}=\left[q_{1 e}, q_{2 e}, q_{3 e}\right]^{T}$ is defined as $\bar{q}_{e}=\bar{q}_{c}^{-1} \otimes \bar{q}$ where $\otimes$ represents quaternion multiplication such that $R\left(\bar{q}_{e}\right)=R\left(\bar{q}_{c}\right)^{T} R(\bar{q})$ then

$$
\dot{R}\left(\bar{q}_{e}\right)=\dot{R}\left(\bar{q}_{c}\right)^{T} R(\bar{q})+R\left(\bar{q}_{c}\right)^{T} \dot{R}(\bar{q}),
$$

which gives

$$
\dot{R}\left(\bar{q}_{e}\right)=-\Omega_{c} R\left(\bar{q}_{c}\right)^{T} R(\bar{q})+R\left(\bar{q}_{c}\right)^{T} R(\bar{q}) \Omega,
$$

and simplifying yields

$$
\dot{R}\left(\bar{q}_{e}\right)=R\left(\bar{q}_{e}\right)\left(\hat{\Omega}-R\left(\bar{q}_{e}\right)^{-1} \hat{\Omega}_{c} R\left(\bar{q}_{e}\right)\right) .
$$

Defining the relative error velocity as $\hat{\Omega}_{e}=\hat{\Omega}-R\left(\bar{q}_{e}\right)^{-1} \hat{\Omega}_{c} R\left(\bar{q}_{e}\right)$ we can write $\dot{R}\left(\bar{q}_{e}\right)=$ $R\left(\bar{q}_{e}\right) \hat{\Omega}_{e}$ which can be expressed in quaternion form as

$$
\begin{aligned}
& \frac{d \mathbf{q}_{e}}{d t}=\frac{1}{2} \hat{\Omega}_{e} \mathbf{q}_{e}+\frac{1}{2} q_{4 e} \omega_{e} \\
& \dot{q}_{4 e}=-\frac{1}{2} \omega_{e}^{T} \mathbf{q}_{e},
\end{aligned}
$$

where $\omega_{e}=\omega-R\left(\bar{q}_{e}\right)^{T} \omega_{c}$. 


\section{A general quaternion-based tracking control}

In this section a general quaternion-based tracking control is presented with conditions that yield local and global stability of the zero-error state. To prove asymptotic stability the following theorem of Barbashin and Krasovskii is used:

LEMMA 1. Let $x_{e}$ be an equilibrium point of a system $\dot{x}=f(x)$ where $f: D \rightarrow \mathbb{R}^{n}$ is a locally Lipshitz map from a domain $D \subset \mathbb{R}^{n}$ into $\mathbb{R}^{n}$. Let $V: D \rightarrow \mathbb{R}$ be $C^{1}$ on a domain $D$ containing the equilibrium point $x_{e}$, such that $\dot{V}(x) \leq 0$ in $D$. Let $S=\{x \in D: \dot{V}(x)=0\}$ and suppose that no solution can stay identically in $S$, other than the trival solution $x(t) \equiv x_{e}$, then $x_{e}$ is asymptotically stable.

Proof. see Khalil, H. K., (2000).

To track a commanded time-dependent angular velocity $\omega_{c} \in \mathbb{R}^{3}$ and quaternion $\bar{q}_{c} \in$ $\mathbb{S}^{3}$ we define the angular velocity error function as $\omega_{e}=\omega-\omega_{d}$ where $\omega_{d}=R\left(\bar{q}_{e}\right)^{T} \omega_{c}$ with the error quaternion $\bar{q}_{e} \in \mathbb{S}^{3}$ defined by $\dot{q}_{4 e}=-\frac{1}{2} \omega_{e}^{T} \mathbf{q}_{e}$. The zero-error state is defined as $\omega_{e}=[0,0,0]^{T}=\overrightarrow{0}, \bar{q}_{e}=[ \pm 1,0,0,0]$ then for asymptotic stability it is sufficent to show that the tracking error $\left(\omega_{e}, q_{4 e}\right) \in \mathbb{R}^{4}$ converges to one of the equilibrium points in the set $\left(\overrightarrow{0}, q_{4 e}^{*}\right) \in \mathbb{R}^{4}$ where $q_{4 e}^{*}$ can be either 1 or -1 . Let us assume for now that it is sufficient to drive the error to the zero-error state $(\overrightarrow{0}, 1) \in \mathbb{R}^{4}$ then we can define the following general tracking controller

Lemma 2. Let $(\overrightarrow{0}, 1) \in D \subset \mathbb{R}^{4}$ be the zero-error state of the system on a domain $D$. For every solution $\left(\omega_{e}, q_{4 e}\right) \in D$ of the closed-loop system (2.1) and (2.8) with

$$
\boldsymbol{u}=\omega \times J \omega-\sigma J \omega_{e}+k J \frac{\partial H\left(q_{4 e}\right)}{\partial q_{4 e}} \boldsymbol{q}_{e}+J \dot{\omega}_{d}
$$

where $\sigma, k>0$ are scalar constants, then $(\overrightarrow{0}, 1) \in D$ is locally asymptotically stable if the following conditions hold:

(i) $H(1)=0$ with $H\left(q_{4 e}\right)>0$ for $\forall q_{0} \neq 1$

(ii) $H\left(q_{4 e}\right)$ is a $C^{1}$ function with respect to $q_{4 e}$ on $D$

(iii) if $\frac{\partial H\left(q_{4 e}\right)}{\partial q_{4 e}}=0$ on $D$ then its only solution is $q_{4 e}=1$.

Proof. Define a general Lyapunov function on the domain $D$ :

$$
V \equiv \frac{1}{2}\left\langle\omega_{e}, \omega_{e}\right\rangle+2 k H\left(q_{4 e}\right)
$$

where $\langle\cdot, \cdot\rangle$ is the standard inner product, it follows that:

$$
\dot{V}=\left\langle\omega_{e}, \dot{\omega}_{e}\right\rangle+2 k \dot{H}\left(q_{4 e}\right)
$$

then substituting (2.1) in (3.3) gives:

$$
\dot{V}=\left\langle\omega_{e}, J^{-1}(J \omega \times \omega+\mathbf{u})-\dot{\omega}_{d}\right\rangle+2 k \frac{\partial H}{\partial q_{4 e}} \dot{q}_{4 e}
$$

then

$$
\dot{V}=\left\langle\omega_{e}, J^{-1}(-\omega \times J \omega+\mathbf{u})-\dot{\omega}_{d}-k \frac{\partial H}{\partial q_{4 e}} \mathbf{q}_{4 e}\right\rangle
$$

then substituting (3.1) into (3.5) yields:

$$
\dot{V}=-\sigma\left\langle\omega_{e}, \omega_{e}\right\rangle \leq 0
$$

from Lemma 1 for local asymptotic stability we must show that $(\overrightarrow{0}, 1) \in D$ is the only 
solution that can stay identically in the set $S=\left\{\left(\omega_{e}, q_{4 e}\right) \in D: \dot{V}=0\right\}$. Then as $\dot{V}=0$ implies $\omega_{e}=0$ the closed-loop error dynamics on $S$ are:

$$
J \dot{\omega}_{e}=k J \frac{\partial H\left(q_{4 e}\right)}{\partial q_{4 e}} \mathbf{q}_{e} .
$$

with $\dot{q}_{4 e}=\frac{d \mathbf{q}_{e}}{d t}=0$. Then $(\overrightarrow{0}, 1) \in D$ is the only equilibrium point of (3.7) in $S$ if condition (iii) holds. $\square$ There have been several choices of the function $H\left(q_{4 e}\right)$ in the case of both stabilization and tracking and some of these are; (i) $H\left(q_{4 e}\right)=1-q_{4 e}$ in Wie, B., Barba, P. M. (1985), Wie, B., Weiss, H.,Arapostathis, A. (1989), Wen, J. T.,KreutzDelgado, K., (1991), (ii) $H\left(q_{4 e}\right)=\ln \left(1 / q_{4 e}\right)$ in Fjellstad, O. E.,Fossen, T. I., (1994), (iiii) $H\left(q_{4 e}\right)=1-q_{4 e}^{2}$ in Wie, B., Barba, P. M. (1985), Wie, B., Weiss, H.,Arapostathis, A. (1989), Wen, J. T.,Kreutz-Delgado, K., (1991)

Using Lemma 2 it is straightforward to show that the control corresponding to (i) asymptotically stabilises $(\overrightarrow{0}, 1) \in D$ where $D=\left\{\left(\omega_{e}, q_{4 e}\right) \in \mathbb{R}^{4}: q_{4 e} \in(-1,1]\right\}$, that is, $H(1)=0$ and $H\left(q_{4 e}\right)>0$ for all values $q_{0} \neq 0$ with $(\overrightarrow{0}, 1) \in D$ the largest invariant set in $S=\left\{\left(\omega_{e}, q_{4 e}\right) \in D: \omega=0\right\}$. However, $(\overrightarrow{0},-1) \in D$ corresponds to the same zero-error state but is unstable. Starting at this unstable zero-error state will cause the system to rotate unnecessarily until it reaches the stable zero-error state $(\overrightarrow{0}, 1) \in D$. This effect is known as the unwinding problem see Bhat,S. P., Bernstein D, S., (2000). The control law corresponding to (iii) avoids the unwinding problem associated with the other control laws as both $(\overrightarrow{0}, 1) \in D$ and $(\overrightarrow{0},-1) \in D$ are locally asymptotically stable but has an unstable equilibrium point of the closed-loop system at $q_{4 e}=0$. Moreover, it is well known that it is impossible to have a continuous control law that stabilises the zero-error state $(\overrightarrow{0}, \pm 1) \in D$ where $D$ is the entire error domain, see Bhat,S. P., Bernstein D, S., (2000).

In order to design a control that avoids unwinding it is useful to define the notion of set stability as in Fragopoulos, D., Innocenti, M., (2004) but generalising it to the tracking problem. Denote the desired quaternion error set by $q_{4 e}^{*} \in\{-1,1\}$ and the desired tracking error state by the set $E=\left\{\left(\omega_{e}, q_{4 e}^{*}\right) \in D: \omega_{e}=\overrightarrow{0}, q_{4 e}^{*} \in\{-1,1\}\right\}$. Let $\left(\omega_{e}, q_{4 e}\right) \in D$ and define:

$$
B\left(r_{0}, E\right) \equiv\left(\underset{\left(0, q_{4 e}^{*}\right) \in E}{\cup} B\left(r_{0},\left(\omega_{e}, q_{4 e}\right)\right)\right) \cap D
$$

where $B\left(r_{0},\left(\omega_{e}, q_{4 e}\right)\right) \equiv\left\{\left(\omega_{e}, q_{4 e}\right):\|\omega\|+\left\|q_{4 e}-q_{4 e}^{*}\right\|<r_{0}\right\}$ and define set positive definite function $V$, as $V\left(\omega_{e}, q_{4 e}\right)>0 \forall\left(\omega_{e}, q_{4 e}\right) \in D,\left(\omega_{e}, q_{4 e}\right) \notin E$ and $V(E)=0$.

DEFinition 1. The desired equilibrium set $E$ of the closed-loop error system, is said to be stable if, for any $r_{0}>0$, there exists an $r>0$ such that if $\left(\omega_{e}(0), q_{4 e}(0)\right) \in B(r, E)$ then $\left(\omega_{e}(t), q_{4 e}(t)\right) \in B\left(r_{0}, E\right)$ for all $t \geq 0$. The set $E$ is asymptotically stable if it is stable and there exists an $r>0$ such that for $\left(\omega_{e}(0), q_{4 e}(0)\right) \in B(r, E)$ implies that $\min _{\left(\overrightarrow{0}, a_{*}^{*}\right) \in E}\|\omega\|+\left\|q_{4 e}-q_{4 e}^{*}\right\| \rightarrow 0$ as $t \rightarrow 0$ and it is globally asymptotically stable if it is $\left(\overrightarrow{0}, q_{4 e}^{*}\right) \in E$ asymptotically stable for any given state.

Defining the sets $A_{1}=\left\{\left(\omega_{e}, q_{4 e}\right) \in D: \omega_{e} \in \mathbb{R}^{3}, q_{4 e} \in[0,1]\right\}$ and $A_{2}=\left\{\left(\omega_{e}, q_{4 e}\right) \in D\right.$ : $\left.\omega_{e} \in \mathbb{R}^{3}, q_{4 e} \in[-1,0)\right\}$ such that $A_{1} \cap A_{2}=\emptyset$ we state the following theorem

Theorem 1. Let $D=A_{1} \cup A_{2}$ be the entire error domain, with tracking error $\left(\omega_{e}, q_{4 e}\right) \in$ $D$. Then for any initial tracking error $\left(\omega_{e}(0), q_{4 e}(0)\right) \in A_{i}$ the control law:

$$
\boldsymbol{u}=\omega \times J \omega-\sigma J \omega_{e}+k J \frac{\partial H_{i}\left(q_{4 e}\right)}{\partial q_{4 e}} \boldsymbol{q}_{e}+J \dot{\omega}_{d}
$$


where $i=1$ if $q_{4 e}(0) \in[0,1]$ and $i=2$ if $q_{4 e}(0) \in[-1,0)$ where $\sigma, k>0$ are positive scalar constants, the equilibrium set $E$ is globally asymptotically stable on $D$ if the following conditions hold:

(i) $H_{1}(1)=0$ and $H_{1}\left(q_{4 e}\right)>0$ for $q_{4 e} \neq 1$ on $A_{1}$ and $H_{2}(-1)=0$ and $H_{2}\left(q_{4 e}\right)>0$ for $q_{4 e} \neq-1$ on $A_{2}$

(ii) $H_{1}\left(q_{4 e}\right)$ is a $C^{1}$ function with respect to $q_{4 e}$ on $A_{1}$ and $H_{2}\left(q_{4 e}\right)$ is a $C^{1}$ function with respect to $q_{4 e}$ on $A_{2}$

(iii) if $\frac{\partial H_{1}\left(q_{4 e}\right)}{\partial q_{4 e}}=0$ on $A_{1}$ then the only solution is $q_{4 e}=1$ and if $\frac{\partial H_{2}\left(q_{4 e}\right)}{\partial q_{4 e}}=0$ on $A_{2}$ its only solution is $q_{4 e}=-1$.

Proof.

Defining the piecewise Lyapunov function on $D=A_{1} \cup A_{2}, A_{1} \cap A_{2}=\emptyset$ with $(\overrightarrow{0}, 1) \in A_{1}$ and $(\overrightarrow{0},-1) \in A_{2}$ :

$$
V=\left\{\begin{array}{lll}
\frac{1}{2}\left\langle\omega_{e}, \omega_{e}\right\rangle+2 k H_{1}\left(q_{4 e}\right) & \text { if } \quad\left(\omega, q_{4 e}\right) \in A_{1} \\
\frac{1}{2}\left\langle\omega_{e}, \omega_{e}\right\rangle+2 k H_{2}\left(q_{4 e}\right) & \text { if } \quad\left(\omega, q_{4 e}\right) \in A_{2}
\end{array}\right.
$$

the proof of conditions (i) and (ii) follow from an application of Lemma 2 applied seperately to each domain $A_{1}$ and $A_{2}$. For (iii) note the sets $S_{1}=\left\{\left(\omega_{e}, q_{4 e}\right) \in A_{1}: \omega_{e}=\overrightarrow{0}\right\}$ and $S_{2}=\left\{\left(\omega_{e}, q_{4 e}\right) \in A_{2}: \omega_{e}=\overrightarrow{0}\right\}$ imply that the closed-loop dynamics on $S_{1}$ and $S_{2}$ are:

$$
J \dot{\omega}_{e}=k J \frac{\partial H_{i}\left(q_{4 e}\right)}{\partial q_{4 e}} \mathbf{q}_{e}
$$

where $i=1,2$ respectively so if $\frac{\partial H_{i}\left(q_{4 e}\right)}{\partial q_{4 e}}=0$ does exist then (iii) must hold.

An example of a tracking controller of the class described in Theorem 1 is $H_{1}\left(q_{4 e}\right)=$ $1-q_{4 e}$ and $H_{2}\left(q_{4 e}\right)=1+q_{4 e}$. It is conveneient to write this in the form $H_{i}\left(q_{4 e}\right)=$ $1-\operatorname{sgn}(i) q_{4 e}$ with $i=1,2$ and where

$$
\operatorname{sgn}(i)=\left\{\begin{array}{ccc}
1 & \text { for } & i=1 \\
-1 & \text { for } & i=2
\end{array}\right.
$$

THEOREM 2. The magnitude of the principal rotation angle tracking error $\theta_{e}$ for the tracking control (3.9) is given by the equation:

$$
\ddot{\theta}_{e}+\sigma \dot{\theta}_{e}+\operatorname{sgn}(i) 2 k \frac{\partial H_{i}\left(\theta_{e}\right)}{\partial \theta_{e}}=0
$$

Proof. the closed-loop dynamics for the general quaternion feedback control (3.9) can be stated as:

$$
\dot{\omega}_{e}=-\sigma \omega_{e}+k \frac{\partial H_{i}\left(q_{4 e}\right)}{\partial q_{4 e}} \mathbf{q}_{e}
$$

assuming an eigen-axis rotation

$$
\begin{aligned}
& \mathbf{q}_{e}(t)=c \mathbf{q}_{e}(0) \\
& \omega_{e}(t)=v \mathbf{q}_{e}(0)
\end{aligned}
$$

where $c(0)=1$ then $(3.14)$ reduces to:

$$
\dot{v}=-\sigma v+k \frac{\partial H_{i}\left(q_{4 e}\right)}{\partial q_{4 e}} c
$$

and noting that the vectors $\mathbf{q}_{e}(t)$ and $\omega_{e}(t)$ are parallel and therefore $\mathbf{q}_{e}(t) \times \omega_{e}(t)=0$ 
and therefore $\hat{\Omega}_{e} \mathbf{q}_{e}=0$ the kinematics (2.8) reduce to:

$$
\begin{gathered}
\dot{c}=\frac{1}{2} q_{4 e} v \\
\dot{q}_{4 e}=-\frac{1}{2} c v\left(1-q_{4 e}(0)^{2}\right)
\end{gathered}
$$

where $q_{4 e}(0)$ is the initial error in the scalar part of the quaternion. Note that independently of the choice of $H_{i}\left(q_{4 e}\right)$ we can define a conservation law from equations (3.17) and (3.18), that is:

$$
\frac{d c}{d q_{4 e}}=-\frac{q_{4 e}}{c\left(1-q_{4 e}^{2}(0)\right)}
$$

and therefore

$$
-\int c d c=\frac{1}{\left(1-q_{4 e}^{2}(0)\right)} \int q_{4 e} d q_{4 e}
$$

and with $c(0)=1$ reveals the conserved quantity that implicitly defines an ellipse:

$$
1=q_{4 e}^{2}+\left(1-q_{4 e}^{2}(0)\right) c^{2}
$$

this form of the conservation law suggests a useful parameterization of this system:

$$
q_{4 e}=\operatorname{sgn}(i) \cos \frac{\theta_{e}}{2}, c=\frac{\sin \theta_{e} / 2}{\sin \left(\theta_{e}(0) / 2\right)}
$$

differentiating these with respect to time and using the chain rule $\frac{\partial H_{i}}{\partial q_{4 e}}=\frac{\partial H_{i}}{\partial \theta_{e}} \cdot \frac{\partial \theta_{e}}{\partial q_{4 e}}$ where $\frac{\partial \theta_{e}}{\partial q_{4 e}}=-\operatorname{sgn}(i) \frac{2}{\sin \theta_{e} / 2}$ the reduced kinematics can be expressed as:

$$
\begin{aligned}
& \dot{v}=-\sigma v-\operatorname{sgn}(i) \frac{2 k}{\sin \left(\theta_{e}(0) / 2\right)} \frac{\partial H_{i}(\theta)}{\partial \theta_{e}} \\
& \dot{\theta}_{e}=\sin \left(\theta_{e}(0) / 2\right) v
\end{aligned}
$$

which on differentiation with respect to time reduces to (3.13). $\square$ In the classical example of Wie, B., Barba, P. M. (1985) where $H_{i}\left(q_{0 e}\right)=1-\operatorname{sgn}(i) q_{4 e}$ so $H_{i}\left(\theta_{e}\right)=$ $1-\operatorname{sgn}(i) \cos \left(\theta_{e} / 2\right)$ the equations (3.13) reduce to the nonlinear damped pendulum:

$$
\ddot{\theta}_{e}+\sigma \dot{\theta}_{e}+k \sin \left(\theta_{e} / 2\right)=0 .
$$

The procedure to tune the controller in Wie, B., Barba, P. M. (1985) is then to linearise the non-linear equations (3.24) and select the parameters for critical damping. However, this approximation is only suitable for small $\theta_{e}$ and for large errors a larger value of the parameter is required to compensate for the non-linear term.

\section{A special case}

The intuition behind this quaternion-based control law stems from both an associated control-Lyapunov function defined on the rotation group Biggs, J. D., Horri, N., (2014) and a control-Lyapunov function defined in terms of the angular velocity error and the eigen-angle error $\theta_{e}$ where

$$
V \equiv \frac{1}{2}\left\langle\omega_{e}, \omega_{e}\right\rangle+k_{1} \theta_{e}^{2}
$$

where $\langle\cdot, \cdot\rangle$ is the standard inner product and $k_{1}$ is a constant. To obtain a Lyapunov function in its corresponding quaternion form the substitution $\theta_{e}=2 \arccos \left(\operatorname{sgn}(i) q_{4 e}\right)$ 
EXAMPLE: A LARGE ANGLE SLEW MANEUVER FOR A SPACECRAFT 7

into (4.1) can be made to yield the Lyapunov function

$$
V \equiv \frac{1}{2}\left\langle\omega_{e}, \omega_{e}\right\rangle+2 k \arccos ^{2}\left(\operatorname{sgn}(i) q_{4 e}\right)
$$

Thus, from Theorem 1 we have $H_{i}\left(q_{0 e}\right)=\arccos ^{2}\left(\operatorname{sgn}(i) q_{4 e}\right)$ which leads to the following globally stabilising feedback tracking control:

$$
\mathbf{u}=\omega \times J \omega-\sigma J \omega_{e}-\operatorname{sgn}(i) 2 k J \frac{\cos ^{-1}\left(\operatorname{sgn}(i) q_{4 e}\right)}{\sqrt{1-q_{4 e}^{2}}} \mathbf{q}_{e}+J \dot{\omega}_{d},
$$

where $k>0$ a constant scalar. It follows from Theorem 2 that the closed-loop error system can be reduced to the description of a linear oscillator:

$$
\ddot{\theta}_{e}+\sigma \dot{\theta}_{e}+k \theta_{e}=0
$$

note that for critical damping we can set $\sigma=2 \sqrt{k}$ and then

$$
\theta_{e}=\theta_{0 e} e^{-\sqrt{k} t}
$$

Thus, $\theta_{e}$ converges to zero exponentially fast, with a critically damped response without oscillation. Note that the linear equation (4.4) is derived without the need for linearization.

\section{Example: a large angle slew maneuver for a spacecraft}

In the following section we compare the proposed tracking control law to a quaternion feedback regulator for spacecraft eigenaxis rotations Wie, B., Weiss, H.,Arapostathis, A. (1989). We consider applying the proposed control to perform a simple large angle attitude manoevre so that a direct comparison can be made with the conventional control law in Wie, B., Weiss, H.,Arapostathis, A. (1989). Assuming the final desired velocity is $\omega_{d}=0$ and thus $\omega_{e}=\omega$ then the control (4.3) reduces to Control B:

$$
\mathbf{u}=\omega \times J \omega-\sigma J \omega-\operatorname{sgn}(i) 2 k J \frac{\cos ^{-1}\left(\operatorname{sgn}(i) q_{4 e}\right)}{\sqrt{1-q_{4 e}^{2}}} \mathbf{q}_{e} .
$$

This can be compared directly to the quaternion feedback regulator for eigneaxis rotations Wie, B., Weiss, H.,Arapostathis, A. (1989) which we call Control A:

$$
\mathbf{u}=\omega \times J \omega-\sigma J \omega-\operatorname{sgn}(i) k J \mathbf{q}_{e} .
$$

A simple tuning method for the quaternion feedback regulator $\sigma^{2}=4 k$ is used. This allows us to compare the effect of the different quaternion error terms in the control laws in (5.1) and (5.2) as all other terms are then equal. The performance of the control (5.1) against (5.2) is undertaken in simulation of a micro-spacecraft with moments of inertia $\left(\mathrm{kg} / \mathrm{m}^{2}\right)$

$$
J=\left(\begin{array}{ccc}
19 & 0.41 & 0.44 \\
0.41 & 19.5 & -0.46 \\
0.44 & -0.46 & 12.6
\end{array}\right)
$$

which is typical of a $100 \mathrm{~kg}$ class satellite. This class of micro-satellite can realistically be equipped with reaction wheels having up to a $100 \mathrm{mNm}$ torque capability and this actuator constraint is included in the simulation. Simulations are undertaken for a constant reference motion of $\bar{q}_{c}=[1,0,0,0]^{T}$ and $\omega_{c}=[0,0,0]^{T}$ with initial conditions $\bar{q}_{e}(0)=\left[1.2 \times 10^{-6}, 0.57735,0.57735,0.57735\right]^{T}$ and $\omega_{e}=[0,0,0]^{T}$. The convergence time is taken to be complete when all components of the angular velocity and quaternion error are of the order $10^{-5}$ for the first time. Figure 1 shows the convergence times for the 


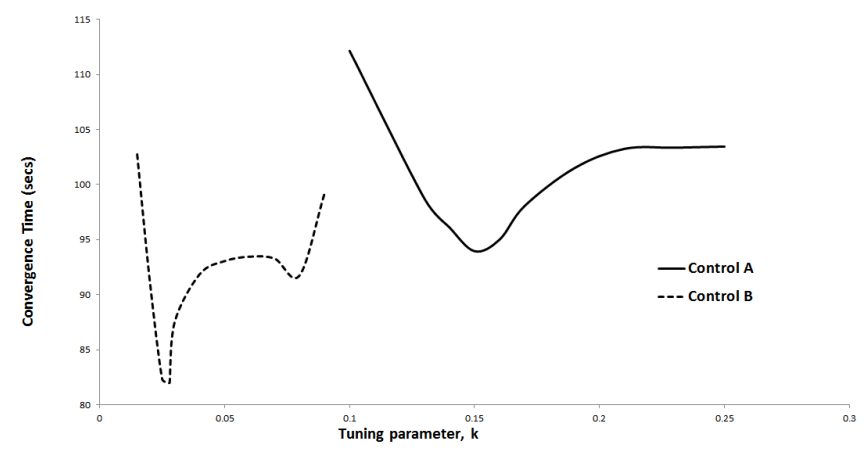

Figure 1. Convergence times for the eigenaxis regulator Control A (5.2) and the Control B

presented tracking control and the quaternion feedback regulator for spacecraft eigenaxis rotations for various values of the tuning parameterk. For this example motion the convergence times are demonstrated to be faster for control (5.1) than (5.2).

\section{Conclusions}

A quaternion tracking controller has been proposed that tracks a desired reference trajectory exponentially fast and without oscillation for any intial error. Simulations show that for the proposed control there is an improvement in settling time for a large angle rest-to-rest motion of a spacecraft with realistic actuator constraints when compared to a more conventional proportional quaternion controller.

\section{REFERENCES}

Wie, B., Weiss, H.,Arapostathis, A. 1989 Quaternion feedback regulator for spacecraft eigenaxis rotations. Journal of Guidance 12, 375-380.

Wen, J. T.,Kreutz-Delgado, K. 1991 The attitude control problem. IEEE Transactions on Automatic control. 36, 1148-1162.

Wie, B., BARBA, P. M. 1985 Quaternion feedback for spacecraft large-angle manoeuvres. Journal of Guidance, Control and Dynamics. 8, 360-365.

FJellstad, O. E.,Fossen, T. I., 1994 Quaternion feedback regulation of Underwater Vehicles Proceedings of the 3rd IEEE Conference on Control Applications 2, 857862.

Tayebi, A., McGilvray, S., 2006 Attitude Stabilization of a VTOL Quadrotor Aircraft IEEE Transactions on Control systems technology 14, 562571.

Bhat,S. P., Bernstein D, S., 2000 A topological obstruction to continuous global stabilization of rotational motion and the unwinding phenomenon Systems \& Control Letters. 39,6370.

Fragopoulos, D., InNocenti, M. 2004 Stability considerations in quaternion attitude control using discontinuous Lyapunov functions IEE Proc.- Control Theory Appli. 151, 253258.

Hughes, P. C., 2004 Spacecraft Attitude Dynamics, Dover Books on Aeronautical Engineering.

Khalil, H. K., 2000 Nonlinear Systems, Prentice-Hall.

Biggs, J. D., Horri, N., 2014 An exponentially fast attitude tracking controller on the rotation group Proceedings of the 65th International Astronautical Congress, Toronto. 